\title{
A Correction Method for Measuring Spectral Irradiance of Light Sources Based on Differential Quadrature Method
}

\author{
Yanfei Wang, ${ }^{1}$ Caihong Dai, ${ }^{1}$ Zhifeng Wu, ${ }^{1}$ and Binhua Chen ${ }^{1,2}$ \\ ${ }^{1}$ Division of Optics, National Institute of Metrology, Beijing 100029, China \\ ${ }^{2}$ Beijing Institute of Technology, School of Optoelectronics, Beijing 100081, China \\ Correspondence should be addressed to Yanfei Wang; wangyf@nim.ac.cn
}

Received 19 November 2014; Accepted 4 March 2015

Academic Editor: Ming-Hung Hsu

Copyright (c) 2015 Yanfei Wang et al. This is an open access article distributed under the Creative Commons Attribution License, which permits unrestricted use, distribution, and reproduction in any medium, provided the original work is properly cited.

\begin{abstract}
A novel correction method was demonstrated for measuring spectral irradiance of light sources with a narrow bandwidth. Using the correction method based on differential quadrature method, an estimate of the true value was achieved with measured values of seven adjacent points. The formula of this correction method was derived. Numerical simulations and experimental validation of this correction method were also performed, respectively. This correction method could be used in radiometry, photometry, colorimetry, and other spectrometry fields, especially in the spectrum measurement of LED lamp.
\end{abstract}

\section{Introduction}

Differential quadrature method plays a key role in engineering science and has received intense attention [1-3]. In the area of radiometry, measurement of spectrum with a narrow bandwidth such as light-emitting diode (LED) is a challenging topic. We have obtained ultranarrow spectra with a bandwidth approaching the natural linewidth $[4,5]$. Spectral irradiance is measured by spectroradiometers with a finite bandwidth. The finite bandwidth of spectroradiometers could cause significant errors when the measured light source has a narrow bandwidth compared to that of spectroradiometers [6]. Therefore, it is necessary to apply correction methods to measured data and obtain an estimate of the true value.

In order to satisfy the metrological and industrial demand on bandwidth correction, the International Commission on Illumination (CIE) has set up a technical committee (TC260 ) who is responsible for creating guidelines on bandwidth correction [7, 8]. In 1988, E. I. Stearns and R. E. Stearns demonstrated $S-S$ method [9]. This method is applicable to monochromators with a triangular bandpass function and it requires that the measured wavelength step is equal to the bandwidth. To solve this problem, the differential quadrature approach has been demonstrated, which is applicable to spectrometers with an arbitrary bandpass function and at an arbitrary wavelength step [7]. Several other correction methods have been proposed [10-12].

In the present paper, we present a novel correction method for measuring spectral irradiance of light sources with a narrow bandwidth. The correction method is based on differential quadrature method. Unlike differential quadrature method with three-point formula and five-point formula, we derive seven-point formula theoretically. Using a sine function and a Gaussian function, we validate this correction method through numerical simulations. We also validate seven-point formula experimentally. This correction method could be used in radiometry, photometry, colorimetry, and other spectrometry fields, especially in the spectrum measurement of LED lamp $[9,11,12]$.

\section{Theoretical Calculation}

We present the derivation of seven-point formula in this part. The theoretical calculation is based on differential quadrature method [13]. The true spectrum and the measured spectrum are represented by $S(\lambda)$ and $M(\lambda)$, respectively. The bandpass function of a spectroradiometer is $b(\lambda)$ and the relation between $S(\lambda)$ and $M(\lambda)$ could be written as

$$
M\left(\lambda_{0}\right)=\int S(\lambda) b\left(\lambda-\lambda_{0}\right) d \lambda
$$


where $M\left(\lambda_{0}\right)$ represents measured value at the $\lambda=\lambda_{0}$ point. For monochromators, the bandpass function has a triangular shape in general case, which is shown in Figure 1. The bandwidth is $10 \mathrm{~nm}$ and the bandpass function is normalized. In this paper, we only consider monochromators with a triangular bandpass function.

The true value $S(\lambda)$ could be written in the form of Taylor series expansion around the measured point at the wavelength $\lambda=\lambda_{0}$

$$
S(\lambda)=S\left(\lambda_{0}\right)+\sum_{n=1}^{n=\infty} \frac{1}{n !}\left(\lambda-\lambda_{0}\right)^{n} S^{(n)}\left(\lambda_{0}\right),
$$

in which $S^{(n)}(\lambda)$ means the $n$th order derivative of $S(\lambda)$. Using the notation

$$
I_{n}=\int \lambda^{n} b(\lambda) d \lambda
$$

the measured value could be written as

$$
M\left(\lambda_{0}\right)=I_{0} S\left(\lambda_{0}\right)+I_{1} S^{\prime}\left(\lambda_{0}\right)+\frac{1}{2} I_{2} S^{\prime \prime}\left(\lambda_{0}\right)+\cdots .
$$

From (4), we obtain the true value $S\left(\lambda_{0}\right)$ in the form of measured value and its derivative

$$
\begin{aligned}
S\left(\lambda_{0}\right)= & A_{0} M\left(\lambda_{0}\right)+A_{1} M^{\prime}\left(\lambda_{0}\right)+A_{2} M^{\prime \prime}\left(\lambda_{0}\right) \\
& +A_{3} M^{\prime \prime \prime}\left(\lambda_{0}\right)+A_{4} M^{i v}\left(\lambda_{0}\right)+A_{5} M^{v}\left(\lambda_{0}\right) \\
& +A_{6} M^{v i}\left(\lambda_{0}\right)+\cdots,
\end{aligned}
$$

where the coefficients $A$ are expressed as follows:

$$
\begin{gathered}
A_{0}=1, \quad A_{1}=-I_{1}, \\
A_{2}=I_{1}^{2}-\frac{I_{2}}{2}, \quad A_{3}=-I_{1}^{3}+I_{2} I_{1}-\frac{I_{3}}{6}, \\
A_{4}=I_{1}^{4}-\frac{3 I_{1}^{2} I_{2}}{2}+\frac{I_{3} I_{1}}{3}+\frac{I_{2}^{2}}{4}-\frac{I_{4}}{24}, \\
A_{5}=-I_{1}^{5}+2 I_{1}^{3} I_{2}-\frac{I_{3} I_{1}^{2}}{2}-\frac{3 I_{1} I_{2}^{2}}{4}+\frac{I_{4} I_{1}}{12}+\frac{I_{3} I_{2}}{6}-\frac{I_{5}}{120}, \\
A_{6}=I_{1}^{6}-\frac{5 I_{1}^{4} I_{2}}{2}+\frac{2 I_{1}^{3} I_{3}}{3}+\frac{3 I_{1}^{2} I_{2}^{2}}{2}-\frac{I_{4} I_{1}^{2}}{8}-\frac{I_{1} I_{2} I_{3}}{2} \\
+\frac{I_{5} I_{1}}{60}-\frac{I_{2}^{3}}{8}+\frac{I_{4} I_{2}}{24}+\frac{I_{3}^{2}}{36}-\frac{I_{6}}{720} .
\end{gathered}
$$

In order to obtain the true value, we should calculate each order derivative of the measured spectrum $M(\lambda)$, which is shown as (5). The differentiation theory for an ordinary function is introduced in [13]. A function $f(x)$ is tabulated at equal intervals $h$ of the independent variable $x$. Further notational simplifications are obtained by writing

$$
f(x)=f\left(x_{0}+p h\right)=f\left(x_{p}\right)=f_{p} .
$$

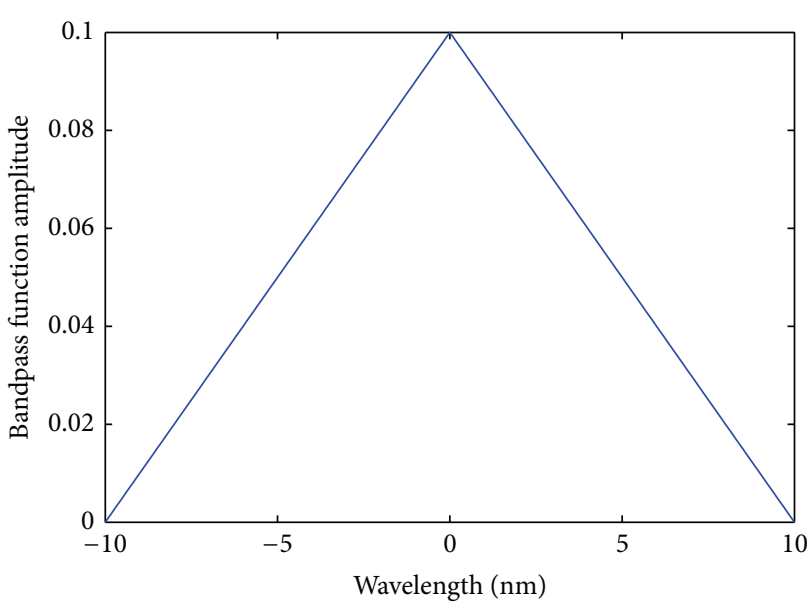

FIGURE 1: A typical bandpass function for monochromators.

The symbol $p$ represents an integer and could be expressed as $p=0, \pm 1, \pm 2, \pm 3, \ldots . f_{0}^{(n)}$ is used to denote the $n$th derivative of $f_{0}$. One has

$$
\begin{gathered}
h f_{0}^{\prime}=\mu \delta_{0}-\frac{1}{6} \mu \delta_{0}^{3}+\frac{1}{30} \mu \delta_{0}^{5}-\cdots \\
h^{2} f_{0}^{\prime \prime}=\delta_{0}^{2}-\frac{1}{12} \delta_{0}^{4}+\frac{1}{90} \delta_{0}^{6}-\cdots \\
h^{3} f_{0}^{\prime \prime \prime}=\mu \delta_{0}^{3}-\frac{1}{4} \mu \delta_{0}^{5}+\frac{7}{120} \mu \delta_{0}^{7}-\cdots \\
h^{4} f_{0}^{i v}=\delta_{0}^{4}-\frac{1}{6} \delta_{0}^{6}+\frac{7}{240} \delta_{0}^{8}-\cdots \\
h^{5} f_{0}^{v}=\mu \delta_{0}^{5}-\frac{1}{3} \mu \delta_{0}^{7}+\frac{13}{144} \mu \delta_{0}^{9}-\cdots \\
h^{6} f_{0}^{v i}=\delta_{0}^{6}-\frac{1}{4} \delta_{0}^{8}+\frac{13}{240} \delta_{0}^{10}-\cdots
\end{gathered}
$$

where $\mu$ is averaging operator and $\delta_{0}^{(n)}$ is $n$th order difference of $f_{0}$.

The displacement operator $E$ is defined as $E f_{p}=f_{p+1}$, using the displacement operator and the following formula:

$$
\begin{gathered}
\delta^{2 n}=E^{-n}(E-1)^{2 n} \\
2 \mu \delta^{2 n+1}=E^{-n-1}\left(E^{2}-1\right)(E-1)^{2 n} .
\end{gathered}
$$

Equation (8) could be written as

$$
\begin{aligned}
h f_{0}^{\prime}= & \frac{1}{2}\left(f_{1}-f_{-1}\right)-\frac{1}{6} \mu \delta^{3} f_{0}+\cdots \\
= & \frac{1}{12}\left(-f_{2}+8 f_{1}-8 f_{-1}+f_{-2}\right)+\frac{1}{30} \mu \delta^{5} f_{0}-\cdots \\
= & \frac{1}{60}\left(f_{3}-9 f_{2}+45 f_{1}-45 f_{-1}+9 f_{-2}-f_{-3}\right) \\
& -\frac{1}{140} \mu \delta^{7} f_{0}+\cdots,
\end{aligned}
$$




$$
\begin{aligned}
h^{2} f_{0}^{\prime \prime}= & \left(f_{1}-2 f_{0}+f_{-1}\right)-\frac{1}{12} \delta^{4} f_{0}+\cdots \\
= & \frac{1}{12}\left(-f_{2}+16 f_{1}-30 f_{0}+16 f_{-1}-f_{-2}\right) \\
& +\frac{1}{90} \delta^{6} f_{0}-\cdots \\
= & \frac{1}{180}\left(2 f_{3}-27 f_{2}+270 f_{1}-490 f_{0}\right. \\
& \left.+270 f_{-1}-27 f_{-2}+2 f_{-3}\right) \\
& -\frac{1}{560} \delta^{8} f_{0}+\cdots, \\
h^{3} f_{0}^{\prime \prime \prime}= & \frac{1}{2}\left(f_{2}-2 f_{1}+2 f_{-1}-f_{-2}\right)-\frac{1}{4} \mu \delta_{0}^{5}+\cdots \\
& -\frac{1}{3} \delta_{0}^{8}+\cdots \delta_{0}^{7}+\cdots, \\
h^{5} f_{0}^{v}= & \frac{1}{2}\left(-f_{3}+8 f_{2}-13 f_{1}+13 f_{-1}-8 f_{-2}+f_{-3}\right) \\
& +\frac{7}{120} \mu \delta_{0}^{7}-\cdots, \\
h^{4} f_{0}^{i v}= & \left(f_{2}-4 f_{1}+6 f_{0}-4 f_{-1}+f_{-2}\right)-\frac{1}{6} \delta_{0}^{6}+\cdots \\
= & \frac{1}{6}\left(-f_{3}+12 f_{2}-39 f_{1}+56 f_{0}\right. \\
& \\
& \left.+12 f_{-2}-f_{-3}\right) \\
& \\
& \\
& \\
&
\end{aligned}
$$

Neglecting the high order differences, each order derivative of the measured spectrum could be written in the form of $M_{p}$. One has

$$
\begin{gathered}
M^{\prime}=\frac{1}{60 h}\left(M_{3}-9 M_{2}+45 M_{1}-45 M_{-1}+9 M_{-2}-M_{-3}\right) \\
M^{\prime \prime}=\frac{1}{180 h^{2}}\left(2 M_{3}-27 M_{2}+270 M_{1}-490 M_{0}\right. \\
\left.\quad+270 M_{-1}-27 M_{-2}+2 M_{-3}\right) \\
M^{\prime \prime \prime}=\frac{1}{8 h^{3}}\left(-M_{3}+8 M_{2}-13 M_{1}\right. \\
\left.\quad+13 M_{-1}-8 M_{-2}+M_{-3}\right) \\
M^{i v}=\frac{1}{6 h^{4}}\left(-M_{3}+12 M_{2}-39 M_{1}+56 M_{0}-39 M_{-1}\right. \\
\left.\quad+12 M_{-2}-M_{-3}\right)
\end{gathered}
$$

$$
\begin{gathered}
M^{v}=\frac{1}{2 h^{5}}\left(M_{3}-4 M_{2}+5 M_{1}-5 M_{-1}+4 M_{-2}-M_{-3}\right), \\
M^{v i}=\frac{1}{h^{6}}\left(M_{3}-6 M_{2}+15 M_{1}-20 M_{0}\right. \\
\left.+15 M_{-1}-6 M_{-2}+M_{-3}\right) .
\end{gathered}
$$

We obtain seven-point formula by neglecting terms after the seventh term in (5). The three-point formula and fivepoint formula could also be obtained by neglecting terms after the third term and the fifth term in (5). For three-point formula, we obtain

$$
\begin{gathered}
S_{3}=a_{-1} M_{-1}+a_{0} M_{0}+a_{1} M_{1}, \\
a_{-1}=-\frac{A_{1}}{2 h}+\frac{A_{2}}{h^{2}}, \\
a_{0}=A_{0}-\frac{2 A_{2}}{h^{2}}, \\
a_{1}=\frac{A_{1}}{2 h}+\frac{A_{2}}{h^{2}},
\end{gathered}
$$

where $S_{n}$ represents $n$-point correction value of the measured point and $a_{n}$ is the coefficient of $M_{n}$.

For five-point formula, we obtain

$$
\begin{gathered}
S_{5}=b_{-2} M_{-2}+b_{-1} M_{-1}+b_{0} M_{0}+b_{1} M_{1}+b_{2} M_{2}, \\
b_{-2}=\frac{A_{1}}{12 h}-\frac{A_{2}}{12 h^{2}}-\frac{A_{3}}{2 h^{3}}+\frac{A_{4}}{h^{4}}, \\
b_{-1}=-\frac{2 A_{1}}{3 h}+\frac{4 A_{2}}{3 h^{2}}+\frac{A_{3}}{h^{3}}-\frac{4 A_{4}}{h^{4}}, \\
b_{0}=A_{0}-\frac{5 A_{2}}{2 h^{2}}+\frac{6 A_{4}}{h^{4}}, \\
b_{1}=\frac{2 A_{1}}{3 h}+\frac{4 A_{2}}{3 h^{2}}-\frac{A_{3}}{h^{3}}-\frac{4 A_{4}}{h^{4}}, \\
b_{2}=-\frac{A_{1}}{12 h}-\frac{A_{2}}{12 h^{2}}+\frac{A_{3}}{2 h^{3}}+\frac{A_{4}}{h^{4}},
\end{gathered}
$$

where $b_{n}$ is the coefficient of $M_{n}$ in five-point formula.

Finally, we obtain seven-point formula by inserting (6) and (11) into (5). One has

$$
\begin{gathered}
S_{7}=c_{-3} M_{-3}+c_{-2} M_{-2}+c_{-1} M_{-1} \\
+c_{0} M_{0}+c_{1} M_{1}+c_{2} M_{2}+c_{3} M_{3}, \\
c_{-3}=-\frac{A_{1}}{60 h}+\frac{A_{2}}{90 h^{2}}+\frac{A_{3}}{8 h^{3}}-\frac{A_{4}}{6 h^{4}}-\frac{A_{5}}{2 h^{5}}+\frac{A_{6}}{h^{6}}, \\
c_{-2}=\frac{3 A_{1}}{20 h}-\frac{3 A_{2}}{20 h^{2}}-\frac{A_{3}}{h^{3}}+\frac{2 A_{4}}{h^{4}}+\frac{2 A_{5}}{h^{5}}-\frac{6 A_{6}}{h^{6}}, \\
c_{-1}=-\frac{3 A_{1}}{4 h}+\frac{3 A_{2}}{2 h^{2}}+\frac{13 A_{3}}{8 h^{3}}-\frac{13 A_{4}}{2 h^{4}}-\frac{5 A_{5}}{2 h^{5}}+\frac{15 A_{6}}{h^{6}}, \\
c_{0}=A_{0}-\frac{49 A_{2}}{18 h^{2}}+\frac{28 A_{4}}{3 h^{4}}-\frac{20 A_{6}}{h^{6}}
\end{gathered}
$$




$$
\begin{gathered}
c_{1}=\frac{3 A_{1}}{4 h}+\frac{3 A_{2}}{2 h^{2}}-\frac{13 A_{3}}{8 h^{3}}-\frac{13 A_{4}}{2 h^{4}}+\frac{5 A_{5}}{2 h^{5}}+\frac{15 A_{6}}{h^{6}}, \\
c_{2}=-\frac{3 A_{1}}{20 h}-\frac{3 A_{2}}{20 h^{2}}+\frac{A_{3}}{h^{3}}+\frac{2 A_{4}}{h^{4}}-\frac{2 A_{5}}{h^{5}}-\frac{6 A_{6}}{h^{6}}, \\
c_{3}=\frac{A_{1}}{60 h}+\frac{A_{2}}{90 h^{2}}-\frac{A_{3}}{8 h^{3}}-\frac{A_{4}}{6 h^{4}}+\frac{A_{5}}{2 h^{5}}+\frac{A_{6}}{h^{6}},
\end{gathered}
$$

where $c_{n}$ is the coefficient of $M_{n}$ in seven-point formula.

\section{Numerical Simulations}

In order to validate seven-point formula, we use a sine function and a Gaussian function to simulate the correction method in this section. We simulate the true spectrum through a function and then obtain the measured spectrum using (1). At last, we apply seven-point formula to measured data. For simplicity, we neglect noise effect in simulation process and experimental validation.

3.1. Sine Function. Firstly, we use a sine function to validate seven-point formula. The expression of the chosen sine function is

$$
y=\frac{1}{2} \sin \left(\frac{\pi}{10} \lambda-\frac{\pi}{2}\right)+\frac{1}{2},
$$

where the wavelength $\lambda$ is the independent variable and $y$ is the amplitude of the spectrum. The period of this function is 20.

Figure 2 shows the simulated measurement and correction of the sine function expressed as (15). For the purpose of comparing the three formulae, bandwidth of the bandpass function is chosen as $B=12$ and the wavelength step $h=0.1$. The true spectrum (solid blue line) is obtained from (15) and the measured spectrum (solid green line) is obtained from (1). We apply three-point formula which is shown as (12) to the measured spectrum and obtain the corrected spectrum (solid black line). Similarly, we could obtain the corrected spectrum using five-point formula (solid yellow line) which is shown as (13) and seven-point formula (solid red line) which is shown as (14). From Figure 2, we note that the measured spectrum is severely distorted compared with the true spectrum. In this particular condition, the measured value at the centre wavelength is $62.7 \%$ of the true value. The percentages for three-point method, five-point method, and seven-point method reach $77.8 \%, 88.5 \%$, and $94.6 \%$, respectively. Therefore, seven-point method does have an obvious correction effect.

The bandwidth determines how effective the correction method would be. We change the bandwidth to $B=8$ and keep other conditions unchanged. The simulated measurement of the sine function and the corrected spectrum with seven-point formula are shown in Figure 3. Similar to that described above, the true spectrum (solid blue line) is obtained from (15) and the measured spectrum (solid green line) is obtained from (1). We apply seven-point formula to the measured spectrum and obtain the corrected

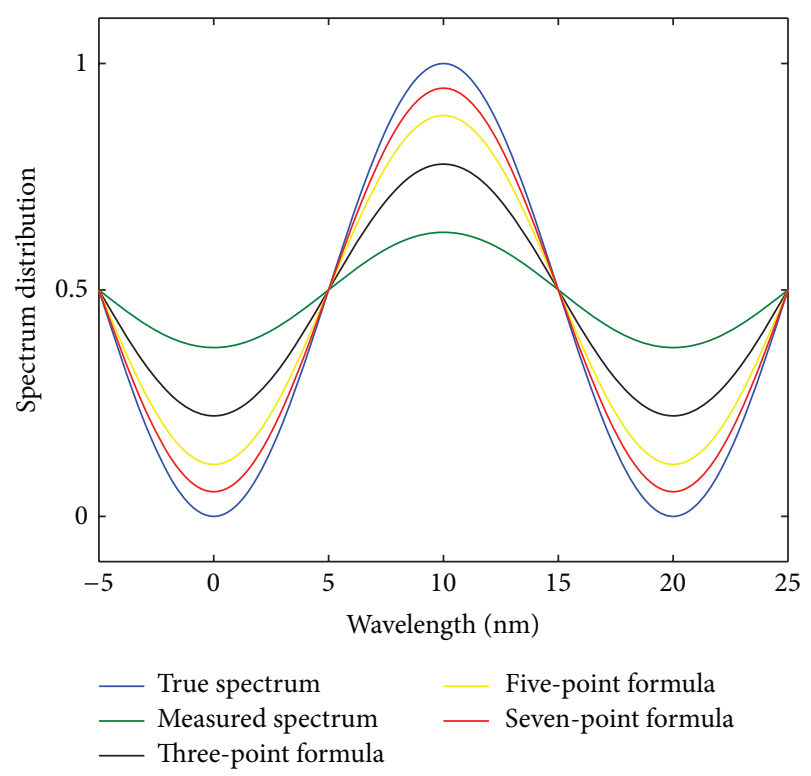

FIGURE 2: The simulated measurement and correction of the sine function. The bandwidth of the bandpass function is $B=12$.

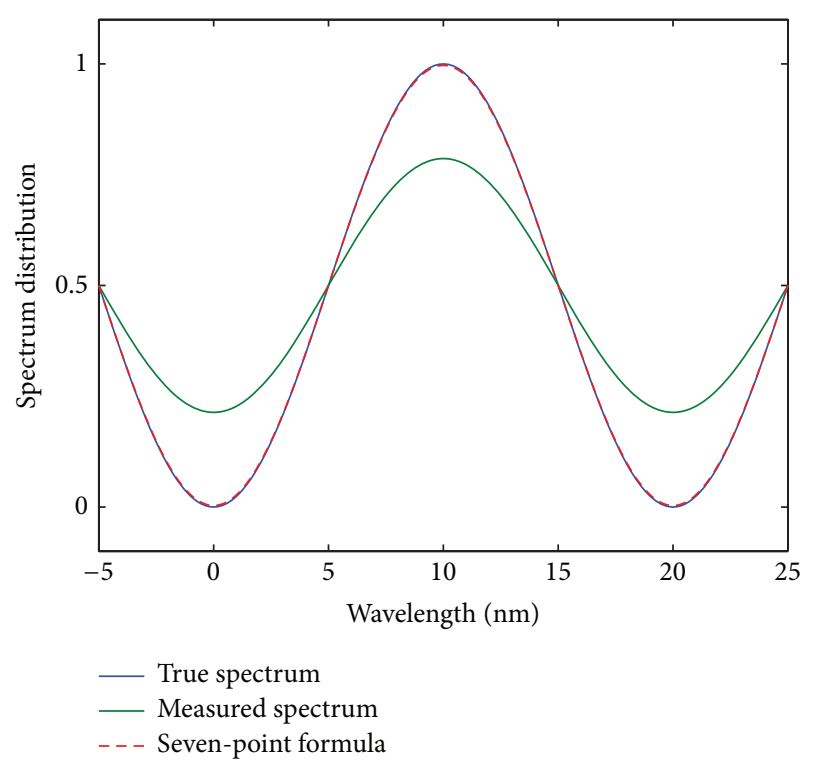

FIGURE 3: The simulated measurement and correction of the sine function. The bandwidth of the bandpass function is $B=8$.

spectrum (dotted red line). The measured value at the centre wavelength is $78.6 \%$ of the true value. After the correction using seven-point method, the percentage reaches $99.7 \%$. The corrected spectrum and the true one almost coincide. With the bandwidth decreasing, the corrected spectrum becomes closer to the true spectrum.

3.2. Gaussian Function. We use a Gaussian function to validate seven-point formula again. The expression of the Gaussian function is

$$
y=e^{-(\lambda-\mu)^{2} / 2 \sigma^{2}}
$$




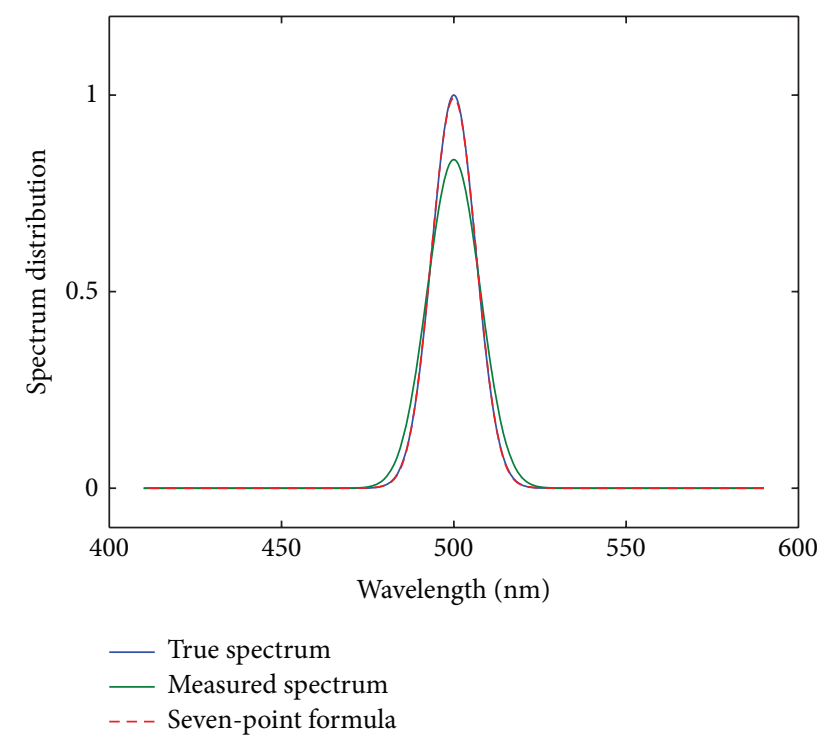

FIgURE 4: The simulated measurement of the Gaussian function and the corrected spectrum with seven-point formula.

where $\mu$ represents the centre wavelength and $\mu=500 \mathrm{~nm}$. The symbol $\sigma$ is $\sigma=\mathrm{FWHM} / \sqrt{8 \ln 2}$. FWHM (Full Width Half Maximum) of this function and the bandwidth are chosen as FWHM $=15 \mathrm{~nm}$ and $B=10 \mathrm{~nm}$.

The simulated measurement of the Gaussian function and the corrected spectrum with seven-point formula are shown in Figure 4. The true spectrum (solid blue line) is obtained from (16) and the measured spectrum (solid green line) is calculated from (1). Seven-point formula is applied to the measured spectrum and corrected spectrum (dotted red line) is obtained. The measured value at the centre wavelength is $83.6 \%$ of the true value. After the correction using sevenpoint method, the percentage reaches $99.3 \%$. Therefore, we validate seven-point formula once again.

\section{Experimental Validation}

In this section, experimental validation of seven-point method is investigated. We measure spectral irradiance of a LED lamp whose centre wavelength is $365 \mathrm{~nm}$ using a double grating spectroradiometer (OL 750D). The bandwidth of this lamp is about $10 \mathrm{~nm}$ and the measured wavelength step is $1 \mathrm{~nm}$. We change the bandwidth of the spectroradiometer by changing width of entrance slit and exit slit. We select proper width of entrance slit and exit slit so that the bandwidth of the spectroradiometer is $B=5 \mathrm{~nm}$ or $B=1 \mathrm{~nm}$. In each case, the spectroradiometer is calibrated and traced to the national primary standard of spectral irradiance, which is based on a high temperature blackbody (HTBB) BB3500M [14]. And then we measure the spectral irradiance when the bandwidth is $5 \mathrm{~nm}$ and $1 \mathrm{~nm}$. The spectroradiometer bandwidth $B=$ $1 \mathrm{~nm}$ is narrow enough compared to the LED bandwidth. Therefore, we consider the measured spectral irradiance at $B=1 \mathrm{~nm}$ as the true spectral irradiance. After that, we apply seven-point formula to the measured spectral irradiance at

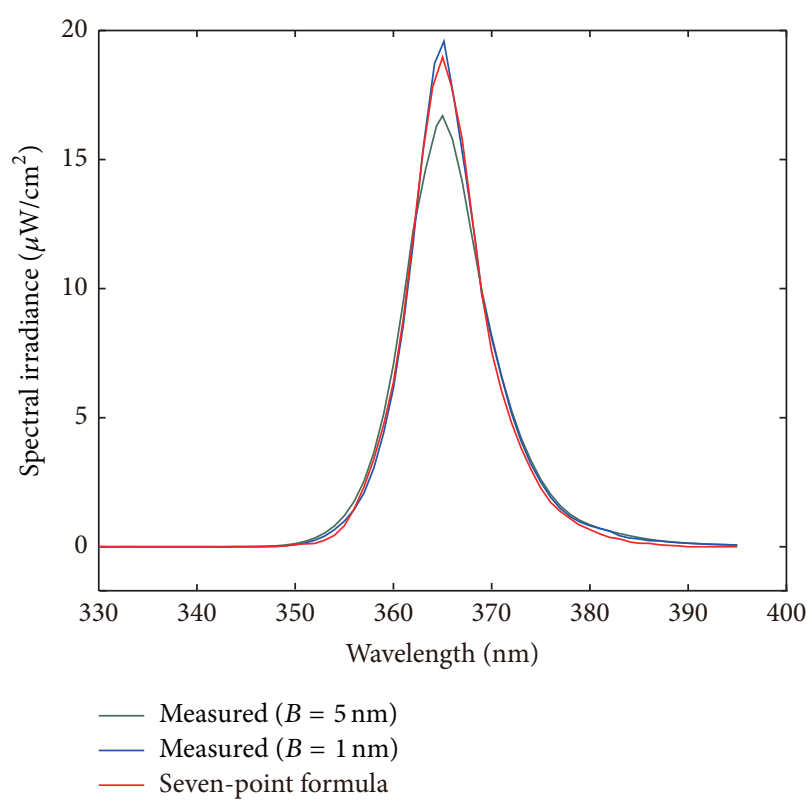

FIgURE 5: The measured spectral irradiance $(B=5 \mathrm{~nm}$ and $B=$ $1 \mathrm{~nm})$ and corrected spectral irradiance $(B=5 \mathrm{~nm})$ with sevenpoint formula.

$B=5 \mathrm{~nm}$ and obtain the corrected spectral irradiance. Finally, we compare the corrected spectral irradiance with the measured one at $B=1 \mathrm{~nm}$. If they are close to each other, it means that the seven-point method is effective.

The experimental results are shown in Figure 5. The peak value of the measured spectral irradiance at $B=5 \mathrm{~nm}$ (solid green line) is $16.7 \mu \mathrm{W} / \mathrm{cm}^{2}$ and the peak value at $B=1 \mathrm{~nm}$ (solid blue line) is $19.4 \mu \mathrm{W} / \mathrm{cm}^{2}$. The ratio between the two values is $86.1 \%$. The peak value of the corrected spectral irradiance for the measured spectral irradiance at $B=5 \mathrm{~nm}$ (solid red line) is $18.9 \mu \mathrm{W} / \mathrm{cm}^{2}$. The ratio between the peak value of the corrected spectral irradiance and that of the measured spectral irradiance at $B=1 \mathrm{~nm}$ is $97.4 \%$. Thus we validate seven-point formula experimentally.

\section{Conclusion}

In conclusion, we have presented a novel correction method for measuring spectra distribution of light sources with a narrow bandwidth. Seven-point formula is derived based on differential quadrature method. We validate this correction method through numerical simulations using a sine function and a Gaussian function. By selecting proper parameters, the corrected value at the centre wavelength could reach above $99 \%$ of the true value. We also validate this correction method experimentally. We measure the spectral irradiance of a LED lamp at $B=5 \mathrm{~nm}$ and $B=1 \mathrm{~nm}$. After that, we apply seven-point formula to the measured spectral irradiance at $B=5 \mathrm{~nm}$ and obtain the corrected spectrum. The ratio between the peak value of the corrected spectral irradiance and that of the measured spectral irradiance at $B=1 \mathrm{~nm}$ is $97.4 \%$. Therefore, we validate the correction method both theoretically and experimentally. 


\section{Conflict of Interests}

The authors declare that there is no conflict of interests regarding the publication of this paper.

\section{Acknowledgments}

The author Yanfei Wang thanks Xiaomeng Li for her assistance in searching the reference [13]. This work was supported by the China Postdoctoral Science Foundation funded project (Grant no. 2014M550788) and the National Natural Science Foundation of China (Grant no. 41305140).

\section{References}

[1] C. W. Bert and M. Malik, "Differential quadrature method in computational mechanics: a review," Applied Mechanics Reviews, vol. 49, no. 1, pp. 1-28, 1996.

[2] C. Shu, H. Ding, and K. S. Yeo, "Local radial basis funcion-based differential quadrature method and its application to solve twodimensional incompressible Navier-Stokes equations," Computer Methods in Applied Mechanics and Engineering, vol. 192, no. 7-8, pp. 941-954, 2003.

[3] Y. L. Chan, L. H. Shen, C. T. Wu, and D. L. Young, "A novel upwind-based local radial basis function differential quadrature method for convection-dominated flows," Computers \& Fluids, vol. 89, pp. 157-166, 2014.

[4] Y. Wang, S. Zhang, D. Wang, Z. Tao, Y. Hong, and J. Chen, "Nonlinear optical filter with ultra-narrow bandwidth approaching the natural linewidth," Optics Letters, vol. 37, no. 19, pp. 40594061, 2012.

[5] Y. Wang, X. Zhang, D. Wang, Z. Tao, W. Zhuang, and J. Chen, "Cs Faraday optical filter with a single transmission peak resonant with the atomic transition at $455 \mathrm{~nm}$," Optics Express, vol. 20, no. 23, pp. 25817-25825, 2012.

[6] H. J. Kostkowski and A. M. Bass, "Slit function effects in the direct measurement of absorption line half-widths and intensities," Journal of the Optical Society of America, vol. 46, no. 12, pp. 1060-1063, 1956.

[7] E. R. Woolliams, R. Baribeau, A. Bialek, and M. G. Cox, "Spectrometer bandwidth correction for generalized bandpass functions," Metrologia, vol. 48, no. 3, pp. 164-172, 2011.

[8] S. Eichstädt, F. Schmähling, G. Wübbeler et al., "Comparison of the Richardson-Lucy method and a classical approach for spectrometer bandpass correction," Metrologia, vol. 50, no. 2, pp. 107-118, 2013.

[9] E. I. Stearns and R. E. Stearns, "An example of a method for correcting radiance data for bandpass error," Color Research \& Application, vol. 13, no. 4, pp. 257-259, 1988.

[10] L.-P. Boivin, "Study of bandwidth effects in monochromatorbased spectral responsivity measurements," Applied Optics, vol. 41, no. 10, pp. 1929-1935, 2002.

[11] S. Nevas, G. Wübbeler, A. Sperling, C. Elster, and A. Teuber, "Simultaneous correction of bandpass and stray-light effects in array spectroradiometer data," Metrologia, vol. 49, no. 2, pp. S43-S47, 2012.

[12] J. L. Gardner, "Bandwidth correction for LED chromaticity," Color Research and Application, vol. 31, no. 5, pp. 374-380, 2006.

[13] Nautical Almanac Office, Interpolation and Allied Tables, HMSO, London, UK, 1956.
[14] C. Dai, Z. Wu, and J. Yu, "Realization of the new national primary scale of spectral radiance and spectral irradiance," in International Symposium on Photoelectronic Detection and Imaging 2013: Imaging Spectrometer Technologies and Applications, vol. 8910 of Proceedings of SPIE, 89100D, p. 8, August 2013. 


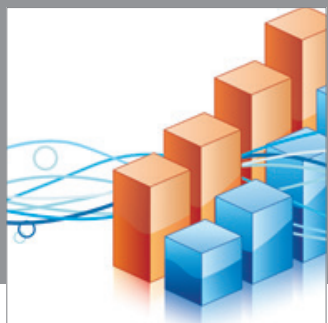

Advances in

Operations Research

mansans

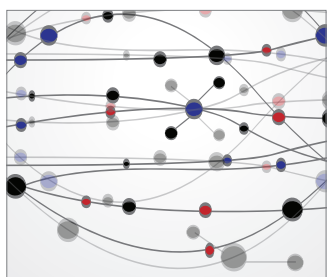

The Scientific World Journal
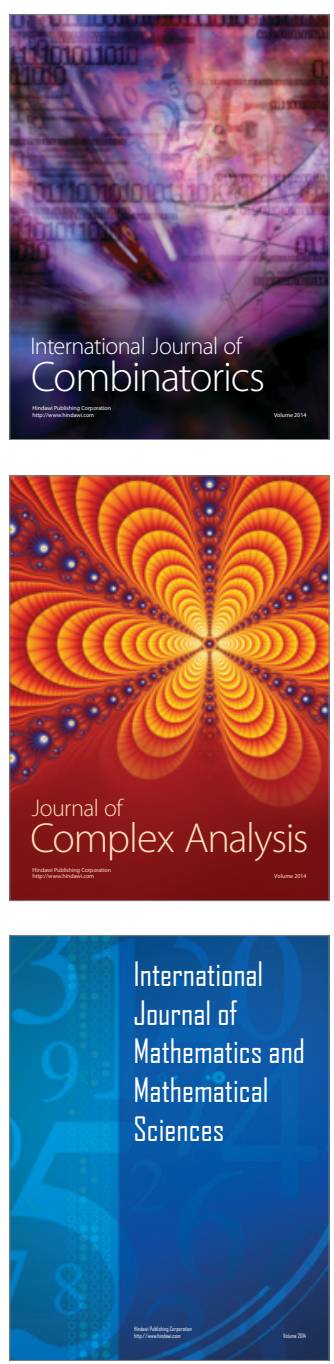
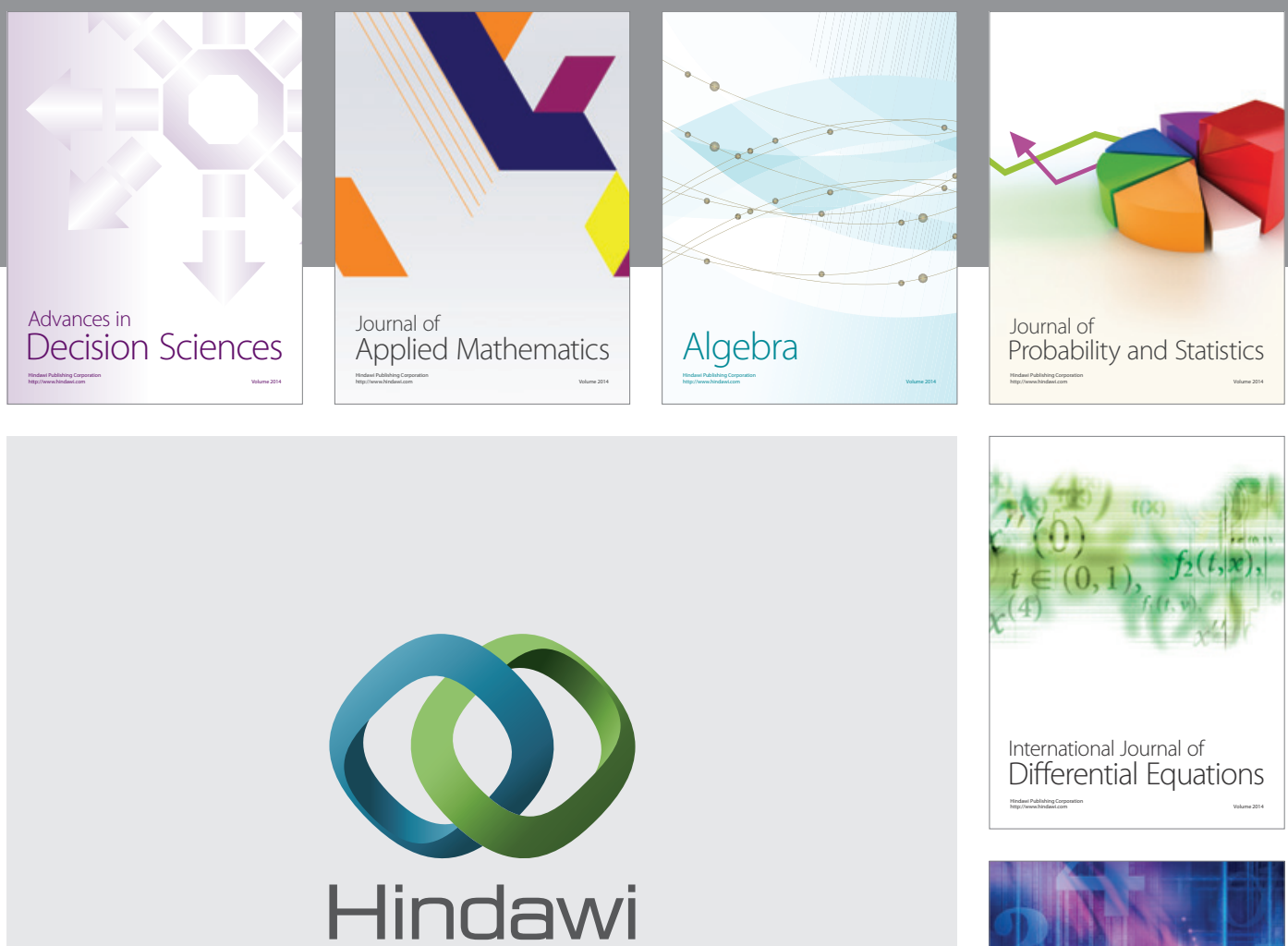

Submit your manuscripts at http://www.hindawi.com
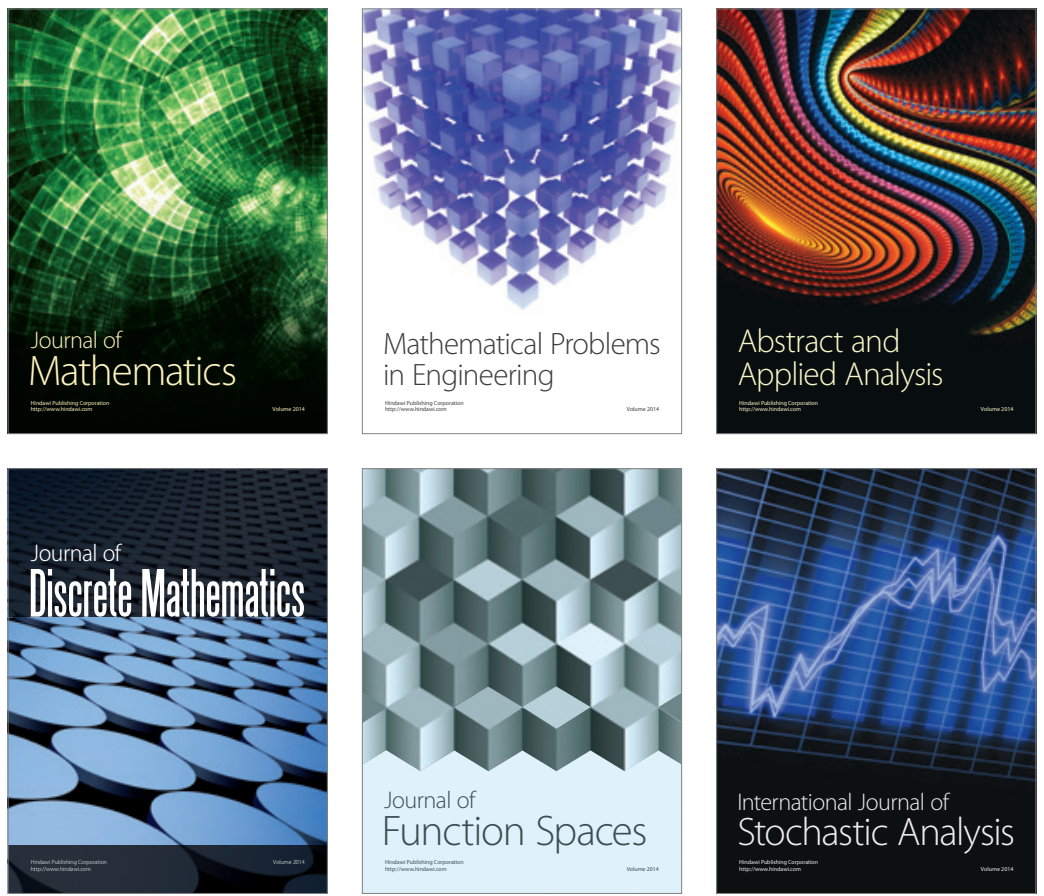

Journal of

Function Spaces

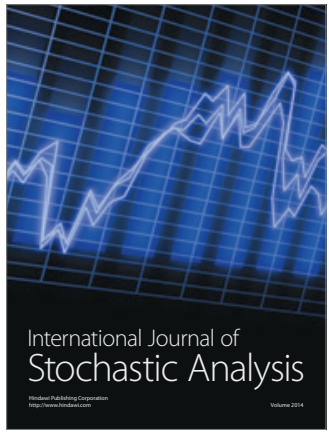

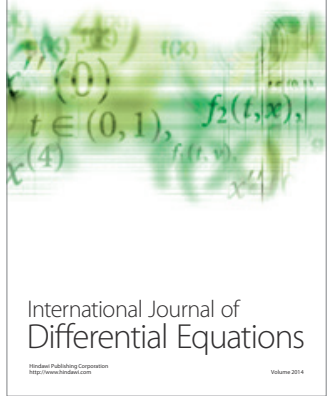
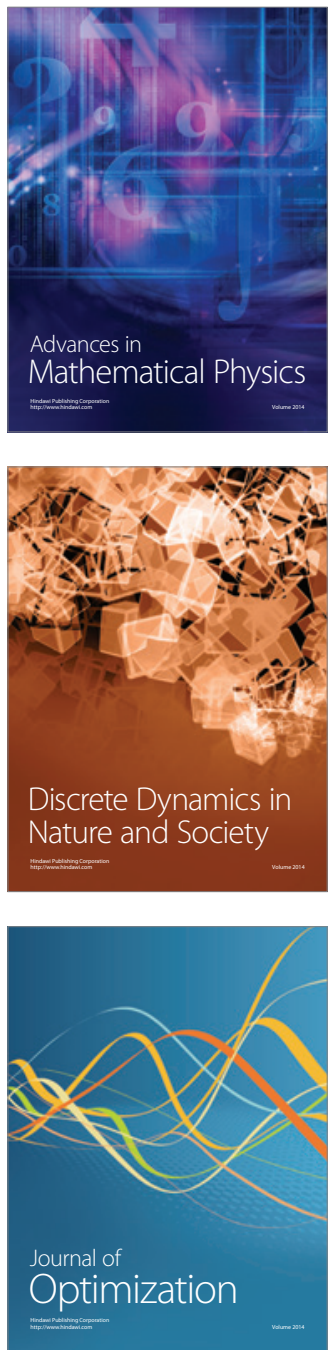